\title{
Contents, Vol. 152, 1966
}

\section{Index}

Avanza, C.: vide Babel, J.

Augustin, M.: Die Bedeutung der Lochlokalisation für die Ablatio-Operation . . 325

Auricchio, G.: vide Diotallevi, M.

Babel, J. et Avanza, C:

Les kystes scléro-cornéens congénitaux et leur evolution

Baumann, H. E.: Optische Korrektur asthenopischer Beschwerden bei Emmetropen mit Hilfe des Polatest 444

Berghoffer, B.: vide O'Rourke, J.

Bider, E.: $\quad$ Zur Kenntnis des «Refsumschen Syndroms» Retinopathia pigmentosa bei hereditärer Enzymopathie des Fettstoffwechsels 356

Black, C. S.: vide Cuendet, J. F.

Bohár, Anna et Sármány, Judit:

Renseignements expérimentaux sur le role du système réticulo-endo-thélial dans les uvéites endogènes 167

Bonting, S. L.: Development and Mode of Action of the Rhodopsin System in the

Photoreceptor Cell 527

Bozin, I.: vide Forgacs, J.

Breitenmoser, R.: Über ein neues Telestereoskop, das Ortho-Telestereoskop oder Orthoskop 118

Broekhuyse, R. M.:

Chemical Aspects of the Juvenile Forms of Lipidosis $\quad 510$

Bruckner, R.: Das Teamwork von Augenarzt und Orthoptistin

Bruijn, J. P. F. M., de:

Clinical Demonstrations $\quad 540$

Cagianut, B. und Schmid, W.:

Mikrophthalmie, Kolobome der Iris und Aderhaut und Analatresie eine neue Chromosomenkrankheit? 354

Collignon-Brach, J.:

vide Weekers, $\mathrm{R}$.

Cuendet, J. F., Black, C. S. et Demiéville, H.:

Possibilités d'automation de Гexamen ophtalmologique 402

Cuendet, J. F.: vide Sévin, R.

Daicker, B.: Zur Kenntnis des Xanthogranuloma juvenile der Lidhaut

Demiéville, H.: vide Cuendet, J. F.

Dieterle, P.: Etat de choc grave après anesthésie locale et son traitement d'urgence 334

Diotallevi, M. and Auricchio, G.:

The Effect of Topically Used Hydergine ${ }^{\circledR}$ on Aqueous Humor Dynamics 193

Draeger, J.: Mikrochirurgisches Vorgehen bei Hornhautoperationen 
Draegeh, J. und Hoffmann, D. H.:

Methodische Überlegungen zur Anwendung von Laser-Geräten zurPhotokoagulation

Eisner, G.: $\quad$ Spaltlampenuntersuchungen der hinteren Augenabschnitte nach intravenöser Fluoreszeininjektion 396

Eisnek, G. und Haldimann, R.:

Beitrag zur Simulationsfrage bei Unfallbegutachtung

Esslen, E. und Jauslin, D.:

Strabismus convergens surso-adductorius und Retraktionsphänomen.Elektromyographische

Analyse eines Falles 466

Faggioni, R.: vide Stucchi, C. A.

Fanta, H. und Obiditsch-Mayer, I.:

Intraoculare Angiogliomatose und Melanoblastom 261

Forgacs, J. et Bozin, I.:

Manifestation familiale de pigmentations groupées de la region macu-

laire $\quad 364$

Francois, J., Szmigielski, M., Rouck, A. de et Hanssens, M.:

Etude électro-physiologique et histologique de la dégénérescence tapé-to-rétinienne

expérimentale par Гiodate de sodium. I. Etude électro-physiologique

Francois, J., Szmigielski, M., Rouck, A. de et Hanssens, M.:

Etude électro-physiologique et histologique de la dégénérescence ta-péto-rétinienne

expérimentale par Гiodate de sodium. II. Etude histologique

Friedmann, A. I.: Serial Analysis of Changes in Visual Field Defects, Employing a

NewInstrument, to Determine the Activity of Diseases Involving the VisualPathways 1

Gailloud, C.: vide Rosselet, E.

Gnädinger, M.: Untersuchungen an Kulturen von Zellen der Kaninchenhornhaut. . 435

Goldmann, H. und Niesel, P.:

Zellzählung in der Vorderkammer $\quad 380$

Grieten, J.: vide Weekers, $\mathrm{R}$.

Hairstone, M. A. and Voe, A. G. de:

Keratomycosis: An Ultrastructural Study

Haldimann, R.: vide Eisner, G.

Hanssens, M.: vide Francois, J.

Hanssens, M.: vide Francois, J.

Heuvel, J. E. A., van den:

Juvenile Lipidosis $\quad 507$

Heuvel, J. E. A., van den:

Clinical Demonstrations

Hilsdorf, C, Marxer, J. und Schönenbergeh, H.:

Beitrag zur Behandlung der postoperativen Chorioidalabhebung . . 294

Hirsch-Hoffmann, A. M.:

Echothiopathe in der Glaukomtherapie 291

Hirsch-Hoffmann, A. M.:

Enzymdiagnostik in der Ophthalmologie 429

Hockwin, O., Okamoto, T., Licht, W. and Noll, E.:

Effect of Pilocarpine on Lens Metabolism 
Hoffmann, D. H.: vide Draeger, J.

Hotz, R.: 2 Fälle mit einseitig erniedrigtem Pulsationsvolumen

Itin, W.: Longueur axiale de $\Gamma æ i l$ chez deux frères atteints de scléro-cornéepériphérique avec cornea plana, Гun présentant une haute myopie,Гautre, une forte hypermétropie (Etude échographique) $\quad 369$

Jauslin, D.: vide Esslen, E.

Keller, H. H.: Kurz-Demonstration eines modifizierten Arruga-Nadelhalters . . . 333

Klöti, R.: $\quad$ Glaskörperchirurgie 303

Körnek, G.: vide Witmer, R.

Kolder, H. and North, A. W.:

Oscillations of the Corneo-Retinal Potential in Animals 149

Lang, J.: Zur Donders'schen Theorie des akkommodativen Schielens .... 453

Leuenberger, A.: Zur konservativen Behandlung des Retinoblastoms 242

Licht, W.: vide Hockwin, 0.

Lund, O.-E.: Combinations of Ocular and Cerebral Malformations with Cranio-facial Dysplasia.

A Clinical, Morphogenetical and HistopathologicalInvestigation

Machemer, R.: vide Schumacher, $H$.

Manschot, W. A.: Congenital Ocular Melanosis, Conjunctival Naevus, Conjunctival Melanosis, Conjunctival Melanoma 495

Martenet, Anne-Catherine:

Uvéite herpétique expérimentale. Reflexions sur un modèle .... 283

Marxer, J.: vide Hilsdorf, C

Mathur, S. P.: Cicatricial Entropion of the upper Eyelid. A Modification in the

Operation 207

Moeschlin, S.: Klinische Demonstration einiger den Ophthalmologen interessierenderSyndrome

(M. Behçet, Arteriitis temporalis Horton, Embolie derArt. centr. retinae nach Herzkatheterismus, maligner Exophthalmusbei Hyperthyreose) 471

Mortada, A.: $\quad$ Lamellar Exfoliation of the Newborn and Ectropion of the Eyelids . $\quad 68$

Niesel, P.: $\quad$ Spaltlampenphotographie der Linse für Meßzwecke 387

Niesel, P.: $\quad$ vide Goldmann, $\mathrm{H}$.

Noll, E.: $\quad$ vide Hockwin, 0.

North, A. W.: vide Kolder, H.

Nouri, A.: $\quad$ Presentation d'un nouvel instrument pour les iridectomies .... 378

Obiditsch-Mayer, I.:

vide Fanta, $\mathrm{H}$.

Okamoto, T.: vide Hockwin, 0.

O'Rourke, J. and Berghoffer, B.:

Uveal Extraction and Clearance of Oxygen in Normal, Inflamed andHyperthermic Eyes 37

Planten, J. T.: Some Queries in Connection with Excision of the Eye for Melano-

blastoma $\quad 490$

Ricci, A.: $\quad$ Emploi du fascia lata dans Topération du ptosis selon Friedenwald

et Guyton 318

Rosselet, E., Gailloud, C. et Verrey, F.:

Retinoblastome et Hypopyon 247

Rouck, A., de: vide Francois, J. 
Rouck, A. de: vide Francois, J.

Sármány, Judit: vide Bohár, Anna

SCHÖNENBERGER, H.:

vide Hilsdorf, $\mathrm{C}$.

Schmid, W.: vide Cagianut, B.

Schumacher, H. und Machemer, R.:

Der Einfluß von Joduracil-desoxyribosid (JDUR) auf die Regeneration

des Hornhautparenchyms 183

Sévin, R. et Cuendet, J. F.:

Effets d'une association d'anthocyanosides de myrtille et de $\beta$-cato.

tène sur la resistance capillaire des diabétiques $\quad 109$

Sinzig, H.: $\quad$ Adaptometrische Prüfung von Pourpranyl Chibret 422

Slooff, J. L. The Rectal Biopsy as a Diagnostic Aid in Atrophia Retinae as Part

of a Systemic Disease 520

Stucchi, C. A. et Faggioni, R.:

Dégénérescence en bandelette primaire et familiale de la cornée . . 343 Szmigielski, M.: vide

Francois, J. Szmigielski, M.: vide Francois, J. Timmerman, G. J. M. E. N.:

The Diagnosis of Tumours in the Region of the Optic Chiasma . . 530 Valk, L. E. M.: Some

Observations on the Treatment of Premature Retinopathy with

Variable Oxygen Tension 506

Velický, J.: Belastungsproben bei der komplizierten Heterochromie (Fuchs) . 161

Verrey, F.: vide Rosselet, E.

Voe, A. G. de: vide Hairstone, M. A.

Weekers, R., Grieten, J. et Collignon-Brach, J.:

Contribution à l'étude de Thypertension oculaire provoquée par la

dexaméthasone dans le glaucome à angle ouvert

81

Werf, P. J. P., van der:

Ocular Pemphigus 547

Witmer, R. und Körner, G.:

Uveitis im Kindesalter

277

Witusik, W.: Types of Physiological Excavation of the Optic Nerve Head .... 57

Zuccoli, A.: Le problème chirurgical de la cataracte associée au glaucome . . 310

Verhandlungsberichte - Proceedings - Congrès

Schweizerische Ophthalmologische Gesellschaft, 58. Generalversamm-lung der Schweizerischen Ophthalmologischen Gesellschaft, Solothurn, vom 16. bis 18. September 1965 - Société Suisse d'Ophtalmologie,58ème Assemblée générale de la Société Suisse d'Ophtalmologie, So-leure, du 16 au 18 septembre $1965 \quad 241,353$

Netherlands Ophthalmological Society, 155th Meeting, UniversityClinic, Nymegen, April 30th and May 1st $1965 \quad 489$

FORTBILDUNG UND OrIENTIERUNG - FURTHER STUDIES AND OTHER

INFORMATION - PER-FECTIONNEMENT ET ORIENTATION

Babel, J.: Rétinopathie due à la chloroquine 74

Buchbesprechungen - Book Reviews - Livres Nouveaux 80

Varia 553

I. Index Rerum 555

Index Autorum 563 
